\title{
BMJ Open Bridging the gap between policy and practice: a qualitative analysis of providers' field experiences tinkering with directly observed therapy in patients with drug-resistant tuberculosis in Addis Ababa, Ethiopia
}

\author{
Kirubel Manyazewal Mussie (D) ,1,2 Christoph Gradmann, ${ }^{1}$ \\ Tsegahun Manyazewal (i) ${ }^{3}$
}

To cite: Mussie KM, Gradmann C, Manyazewal T. Bridging the gap between policy and practice: a qualitative analysis of providers' field experiences tinkering with directly observed therapy in patients with drug-resistant tuberculosis in Addis Ababa, Ethiopia. BMJ Open 2020;10:e35272. doi:10.1136/ bmjopen-2019-035272

- Prepublication history for this paper is available online. To view these files, please visit the journal online (http://dx.doi org/10.1136/bmjopen-2019035272).

Received 29 0ctober 2019 Revised 26 February 2020 Accepted 12 May 2020

\section{Check for updates}

(c) Author(s) (or their employer(s)) 2020. Re-use permitted under CC BY-NC. No commercial re-use. See rights and permissions. Published by BMJ.

For numbered affiliations see end of article.

\section{Correspondence to} Kirubel Manyazewal Mussie; k.m.mussie@studmed.uio.no; manyazewalkirubel@yahoo.com

\section{ABSTRACT}

Objectives Drug-resistant tuberculosis (DR-TB) is one of the major public health threats in low-income countries such as Ethiopia. It is intertwined with larger socioeconomic and political factors that complicate its management and control. Whether directly observed therapy (DOT) is serving its purpose-better patient adherence and treatment outcome-still remains a debatable issue. To contribute to this discussion, this study explored health workers' field experiences tinkering with DOT in patients with DR-TB in Addis Ababa, Ethiopia.

Design A qualitative study using in-depth interviews and focus group discussion.

Setting Ten public healthcare facilities: eight health centres at Addis Ababa Health Bureau level and two TBspecialised hospitals at the Federal Health Bureau level in Ethiopia.

Participants 18 healthcare providers working with DR-TB patients.

Results Three findings emerged from the analysis. First, the purpose of DOT is to ensure that patients go to healthcare facilities and swallow pills under the observation of a healthcare provider. Thus, its rigid application could lead to the emergence of more DR-TB. Second, DOT should be tinkered with and its practice improved by incorporating more counselling and health education, with more flexibility towards, and attentiveness of, patient context. Third, there exists a family-like patientprovider relationship, and providers do understand their patients and empathise with them to provide better healthcare services.

Conclusion If rigidly implemented, DOT could lead to more DR-TB - a problem DOT was invented to resolve. Front-line healthcare providers are sensitive to the tragic experiences of DR-TB patients and empathise with them. Thus, they do not strictly implement DOT and are willing to take any blame resulting from tinkering with it. It is high time to shape the practice of DOT for DR-TB patients, with meaningful contributions from front-line healthcare providers.
Strengths and limitations of this study

- The use of a qualitative methodology was suitable to explore experiences on directly observed therapy.

- The use of two qualitative data collection methods (in-depth interviews and focus group discussion) provided a comprehensive description of concepts.

- Inclusion of study participants from both primaryand tertiary-level healthcare facilities augmented the scope and depth of the study.

- Thematic analysis and an inductive approach to coding facilitated the generation of in-depth accounts of health workers' perceptions and experiences.

- It was not possible to collect data outside of work hours, and this might have affected the chances of having longer discussions with participants.

\section{BACKGROUND}

The global health community has long faced issues interconnected with socioeconomic and political determinants of health. ${ }^{1-6}$ Tuberculosis (TB) is a major infectious disease and a health security threat that the global health community has been struggling to eliminate. ${ }^{7}$ It causes around 10 million people to fall ill and 1.6 million to die every year. ${ }^{8}$ Drug-resistant TB (DR-TB) is a major public health threat and a critical challenge in the prevention and control of TB in many countries. $^{9-11}$ The problem is considerably more complex in sub-Saharan African countries where resources are scarce and political situations are unstable. ${ }^{12-17}$ The WHO 2018 Global TB Report ${ }^{18}$ showed that TB remains a leading cause of death in Africa. The continent accounts for one-quarter of new TB cases and TB deaths worldwide, with 
2.5 million people falling ill and 417000 people dying from TB annually. ${ }^{18}$

The WHO, passing the ambitious End TB Strategy, envisions a world free of TB-zero deaths, disease and suffering due to TB by $2035 .{ }^{19}$ Previous attempts to eradicate TB gave a lesson that a more comprehensive and patient-centred approach is needed to reach such a goal. Pursuant to the 1993 declaration of TB by WHO as a global health emergency, WHO announced the Directly Observed Therapy Short-course (DOTS) - a brand name for the WHO's recommended strategy for TB control by which all countries with a TB problem were to abide. The WHO launched the DOTS in 1995 and it 'became the new mantra. This was what countries needed to integrate into their primary health systems-it was to be the toolkit of their national control programmes'. ${ }^{20}$ The strategy was composed of five distinct elements: political commitment; microscopy services; drug supplies; surveillance and monitoring systems; the use of short-course regimens; and direct observation of treatment. ${ }^{21}{ }^{22}$ However, this TB control strategy had a limitation: it did not take any account of DR-TB and, since the problem of drug resistance in $\mathrm{TB}$ is often linked to poor implementation of DOTS, this rather aggravated the conditions for multidrug-resistant (MDR) TB. ${ }^{23}$ In order to address this gap, DOTS-Plus was built upon the five elements of the DOTS strategy, taking into account the use of second-line anti-TB drugs in MDR-TB endemic settings and thereby aiming to prevent the further development and spread of MDR-TB. ${ }^{24}{ }^{25}$ There are controversies surrounding the feasibility ${ }^{26-28}$ and effectiveness ${ }^{29} 30$ of DOTS-Plus, especially in resource-limited countries. In addition to psychosocial and economic consequences resulting from an approximately 2-year treatment course,$^{30}$ other challenges in implementing DOTS-Plus include the need for more advanced diagnostic tools ${ }^{31}$ and the incidence of adverse events associated with anti-MDR-TB drugs. ${ }^{32}$

The goal of creating a world free of TB relies on, among other measures, ensuring direct observation of drug swallowing-Directly Observed Therapy (DOT). The strict implementation of DOT, especially in sub-Saharan Africa and other resource-limited high-TB burden countries, renders itself vulnerable to critical questions that relate to, for example, human rights and ethics. ${ }^{33}$ In Ethiopia, a country in the horn of Africa with over 100 million population, the national TB programme ${ }^{34}$ complies with the global TB treatment strategy and, therefore, there is no alternative TB treatment strategy to DOT. According to the WHO 2018 report, ${ }^{18}$ TB treatment coverage in Ethiopia is $68 \%$, which is minimum even to accommodate the current DOT needs. The estimated percentage of multidrug- andrifampicin-resistant tuberculosis (MDR/ RR-TB) cases is significantly higher in previously treated cases $(14 \%$ (6.7 to 25$))$ than new cases $(2.7 \%$ (1.6 to $4.1)$ ), which indicates that management of TB treatment under DOT is problematic in the country. There were 680 MDR/RR-TB and four extensively drug-resistant tuberculosis (XDR-TB) laboratory-confirmed cases in the country
Box 1 Summary of the in-depth interviews (IDIs) and focus group discussion (FGD) guides and probes

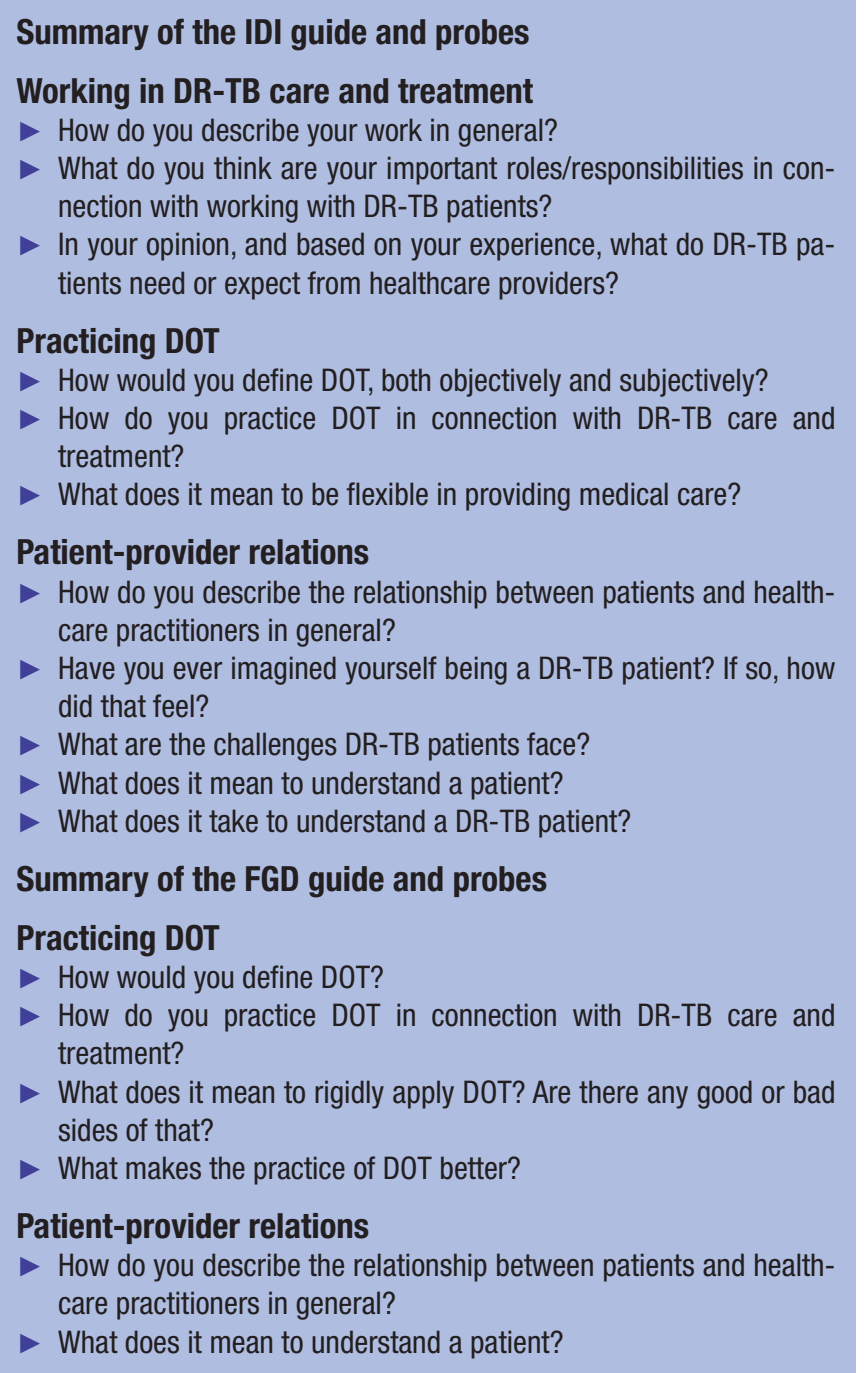

in $2017^{18}$ while many more were presumably left undiagnosed due to limited availability of healthcare services. Whether DOT is serving its purpose-better patient adherence to TB treatment and thereby increased treatment outcome- remains a debatable issue. Thus, this study aimed to explore healthcare providers' perceptions on DOT and their experiences of tinkering with it in the context of DR-TB-'a major threat to public health'. ${ }^{35}$

\section{METHODS}

The study employed a qualitative methodology as the aim of the study was to explore perceptions and experiences. Hence, data were collected through in-depth interviews (IDIs) and focus group discussion (FGD), using interview and FGD guides developed by all authors (Box 1). A total of 18 participants were purposively selected from eight healthcare centres at Addis Ababa Health Bureau level and two TB specialised hospitals at the Federal Health Bureau level in Ethiopia. The two TB-specialised hospitals 
were the only health facilities in Addis Ababa that provide specialised DR-TB diagnosis and care services. The remaining eight healthcare centres were located at varying distances in Addis Ababa, which allowed for potentially different participant characteristics and perceptions. The participants were approached directly, face-to-face after obtaining verbal approval from medical directors at each healthcare facility. Participants were healthcare providers who were employees of the selected healthcare facility at the time of data collection, who took university or college education in health-related discipline, who had worked and/or were working with DR-TB patients at the time of data collection, and who showed their willingness to participate by signing an informed consent form. Healthcare providers who provide direct DR-TB services at the TB clinic, laboratory unit or pharmacy unit were considered eligible. The number of participants was determined by the level of data saturation. ${ }^{36} 37$ The interview was piloted on a clinical nurse to determine the clarity of the questions and to gain experience in conducting an IDI. None of the information from the pretesting was used for the study. Eighteen IDIs were conducted, followed by one FGD consisting of five individual members selected from the IDIs based on their willingness and availability to participate. On average, each IDI took 45 to $55 \mathrm{~min}$ and the FGD lasted 3 hours. Based on consent from participants, all IDIs and the FGD were audio-recorded, with the exception of one participant who preferred that the interviewer would take notes instead of recording.

Data were collected between August and October 2017. The participants themselves decided the place and time to conduct the IDIs and FGD and thus, data were collected in their workplace. The language of communication with the participants was Amharic, which is the working language in the selected healthcare facilities and the mother tongue of the data collector (KMM). As to the impression of the data collector (KMM), the absence of language and cultural barriers helped to build rapport with participants, thereby helping them express themselves more comfortably and openly. ${ }^{38}$ Moreover, the data collector (KMM) reported that the FGD was very lively and the participants were highly concerned about the topic that they wanted to hold a 3-hour discussion, refusing to take a break in between.

In order to ensure anonymity and confidentiality, the participants' names were replaced by pseudonyms. The data collector (KMM) kept the audio files in safe storage where none other could access and deleted them at the end of the study.

\section{Data management and analysis}

We used an inductive approach to analyse the data. Data analysis started during fieldwork so as to identify concepts and gaps early and to continuously explore them in depth throughout the data collection process. We used Braun and Clark's reflexive thematic analysis framework, which involves six phases of analysis-familiarisation, initial coding, theme construction, reviewing themes, defining
Table 1 Demographic characteristics of study participants

\begin{tabular}{lll}
\hline $\begin{array}{l}\text { Name } \\
\text { (anonymised) }\end{array}$ & Sex & Profession/title \\
\hline P1 & Female & Clinical nurse \\
\hline P2 & Female & Clinical nurse \\
\hline P3 & Female & Lab technologist \\
\hline P4 & Male & Health officer \\
\hline P5 & Male & Health officer \\
\hline P6 & Male & Clinical nurse \\
\hline P7 & Male & Health officer \\
\hline P8 & Female & Clinical nurse \\
\hline P9 & Male & Clinical nurse \\
\hline P10 & Female & Clinical nurse \\
\hline P11 & Male & Clinical nurse \\
\hline P12 & Male & Clinical nurse \\
\hline P13 & Female & Clinical nurse \\
\hline P14 & Female & Health officer \\
\hline P15 & Male & Clinical nurse \\
\hline P16 & Male & Clinical nurse \\
\hline P17 & Male & Lab technologist \\
\hline P18 & Male & Clinical nurse \\
\hline & & \\
\hline
\end{tabular}

themes and producing the report. ${ }^{39}$ We analysed the data manually using MS Word. The first author (KMM) openly coded the data line-by-line to identify concepts and to build themes. Trustworthiness-credibility, transferability, dependability and confirmability—was ensured through different ways. The participants were selected based on criteria to ensure that they provide relevant and credible information. All authors were engaged with the data and contributed meaningfully throughout the data analysis. Confirmability was demonstrated by identifying themes and using illustrative quotes.

The data collector (KMM) is a male who studied social work and international community health. During the study period, he was a student taking the master's programme (MPhil) in International Community Health at the University of Oslo, Norway. He received training in qualitative research methods and interview techniques, and has a special interest in critical perspectives in global health.

\section{Patient and public involvement}

Patients and the public were not involved in the design or planning of the study.

\section{RESULTS}

This study included 18 participants (table 1). Findings pertaining to the themes developed from the qualitative data are presented below with thick descriptions-detailed descriptions and interpretations-and illustrative quotes. Three broad themes emerged from the analysis. 


\section{Defining DOT}

This theme discusses healthcare providers' understanding of what DOT is. For the participants, DOT is merely a medical supervision routine in which TB patients swallow pills with the attendance of a healthcare provider: "DOT tells you to go to patients, make them open their mouth and make sure that they swallow the pills." (P13, FGD); "DOT means the patient goes to the health worker until the day he/she finishes the drugs." $(P 7, I D I)$. For the healthcare providers, practicing DOT means nothing more than to see, and make sure, that patients swallow pills. The centre of focus is 'swallowing pills', not patient situation. Due to this, the participants said, there is no counselling in practicing DOT: "DOT doesn't have counselling. DOT and counselling are two different things." (P13, FGD). Unlike defining DOT that seemed to be easy, however, the participants stated that evaluating whether DOT is effective or not is a difficult task. Notwithstanding the rigidity of DOT and the unpleasant experiences it could inflict on patients, as the participants noted, DOT has benefits for both individual patients and the community where they live in: "There are of course some careless patients who must come to us and take their drugs here. For example, if such patients take pills at home, they don't keep the right time and dosage. So in such cases, I support DOT." (P4, FGD); "the patient has an obligation to come daily to the healthcare centre. If the patient refuses to come and quits treatment, who is going to be the looser? It is our community." (P5, FGD). However, on the other hand, some participants during the FGD argued that if rigidly implemented, DOT could lead to more DR-TB-a problem which DOT was invented to fight: "...I think DOT is one cause for the spread of drug resistant TB." (P6, FGD). "This (DOT being one possible cause for the spread of DR-TB) is true. DR-TB patients are aggressive and sensitive due to drug side effects and other reasons. So if you treat your patient according to the DOT principle, like by saying 'take these pills, open your mouth and swallow them', you make things worse." (P13, FGD). Another participant who said that focussing on the DOT task creates a problem echoed this argument: "Patients miss the day when they will finish their drugs and no longer come to us daily. So health workers should be flexible on this. For example, if your patient says 'I am not coming tomorrow because I have a funeral to attend', then you have to give tomorrow's dose for the patient to take it at home. But if you say 'DOT, DOT' and be stubborn, the patient will disappear." (P8, FGD). There was consensus, through both verbal and nonverbal cues, among participants during the FGD that the rigid application of DOT could lead to more drug resistance in TB; that focussing on pill-swallowing burdens patients, pushes them away from TB treatments and makes things worse. Being the most striking result to emerge from the data, this finding, instead of leading to the conclusion that DOT is one of the causes of DR-TB, renders an indication that there is a need to improve the practice of DOT in Ethiopia, so that it serves the purpose it was invented for.
Tinkering and augmenting DOT

Participants in this study emphasised the need to tinker, to compromise and improvise-a medical practice the result of which is an augmented, better DOT practice. The participants argued that it is important to be sensitive to the situation at hand and tinker with care accordingly, instead of committing oneself to rigidly follow DOT: "What I normally do is what my mind tells me. So I just follow my gut feeling. I do not do what is said to be done." (P2, FGD). The healthcare providers see DOT as a treatment strategy that lacks counselling component and, therefore, argue that DOT needs to be tinkered with: "We cannot implement DOT as it is. We give counselling and health education first. So we do not have patients who hide pills, go to the woods and throw them." (P14, IDI). All participants agreed with the statement that good counselling and health education to both DR-TB patients and their relatives should accompany DOT in order to increase its effectiveness. Good counselling, according to the participants, will not only make the practice of DOT more beneficial, but also help to reduce severe psychological consequences among DR-TB patients who suffer from both the disease and its exhaustive treatment: "Imagine how unbearable it is for DR-TB patients to commute to healthcare centres for 2 years. They wish to die than live like this." (P4, IDI); "I had one MDR-TB patient. One day he came to me, looking hopeless and with a very sad face, and said 'for the last 3 days, I have not taken any of those pills you gave me. I have given up and I am ready to die'. This guy fathers two children and has many responsibilities. After much counselling and support from his relatives, it is good that he resumed treatment." $(P 6, I D I)$. It is known that DOT was invented to increase adherence of TB patients to TB treatment and thereby prevent any possible complications. However, the participants noted that DR-TB patients experience treatment fatigue and, as a result, choose to face the consequences of non-adherence. Performing mundane, everyday healthcare tasks, the healthcare providers see the need to negotiate care; to focus on the needs and challenges of the patient whom, after all, the very existence of healthcare is to serve. Therefore, sometimes, healthcare providers give patients the right dosage of DR-TB drugs for them to keep and take at home. Arguing for a patient-centred and understanding-based approach, the participants also mentioned that they are willing to take any blame resulting from flexibly in implementing DOT: "Health workers are blamed for giving patients drugs to take them at home instead of here in front of us. But patients come daily to us and we have to understand that this is tiresome for them. We need to be careful about how we implement DOT. It should not be like a 'command post' (laughter) (The word 'command post' was used to refer to a government body that oversees the state of emergency that was in effect from October 2016 to August 2017 following the public protest against the Ethiopian government. Freedom of public speech, gatherings, access for media, etc were restricted. Using the word 'command post' was common by the public whenever reference to the military force was made). Many people who live in this area have low socioeconomic status and it is unfair to make them come every day and 
take drugs, even though that is what the health authorities tell us to do. So you need to communicate with your patients and understand each other. This is how I have helped many patients to recover. Medication is not a military command, it is rather about an understanding between the patient and the healthcare provider." (P2, IDI).

\section{We are family}

In addition to defining what DOT for them is, and alluding to ways in which the practice of DOT could be improved, the participants reflected on the degree of relationships they have with their patients. Elaborating this, they reported that they understand their patients' situations, see them as friends and families and give them their best. They indicated that the kind of relationship that exists is more than that of between a healthcare provider and a patient: "These patients do not take the drugs because they like them. When I treat my patients, I always imagine myself as a patient. I do my very best, especially on counselling. There was an old female in the age of 50 s who was one of my MDR-TB patients. She didn't not want to follow treatment properly. ... One day, I took off my white coat, sat with her, held her hands and said 'so now, be my mother and listen to what I am saying.' She was happy to hear that and said 'ok my daughter'. So I told her in a way she could understand me, and finally she agreed and said 'God bless you my daughter'. We are family. So we need to understand our patients and give them good counselling." (P2, IDI). All participants mentioned the importance of understanding DR-TB patients in order to increase treatment effectiveness. The inquiry to know to what extent the health workers understand their patients started with an exploration of their empathy for their patients: "I have one MDR-TB patient and giving her injection every day is by itself painful for me. Taking injection even for 1 day is not easy. When that girl comes and says when am I going to take out this mask?' I feel terrible. We know and share the pain of our patients (deep breath and eyes filled with tear)." (P9, IDI); "Whenever I give injection to the patients, I ask myself 'what if I were them?'." (P1, IDI). The healthcare providers reported that they have strong level of empathy and share their patients' pains. Not only do they know the patients' situation and empathise with them, but the participants also said that they feel the pain. Moreover, the participants stated that they face a dilemma in decision-making. In MDR/XDR-TB wards, admitted DR-TB patients are not allowed to freely go out into the community; they are strictly isolated. In a situation such as this, healthcare providers, since they are attentive to their patients' conditions and share their emotional sufferings, find it difficult to comply with the need to strictly isolate patients: "These patients who are admitted here miss the outside world.... If you let them out, they will infect others. What can you do? This dilemma is aching." (P18, IDI). This, in addition to putting them in dilemma to make a decision on whether they should permit patients to, say, go out and buy something, puts the healthcare providers in a stressful situation. However, they did not report whether this has a negative impact on their daily work. The finding here is rather different: looking at patients' suffering shapes the health workers' perception of DR-TB and the way they treat their patients, the effect of which is better and patient-centred treatment: "When you work on DR-TB, you realise that patients and healthcare providers are like families. So you have to work from the bottom of your heart." (P3, IDI).

\section{DISCUSSION}

Our findings indicate that the 'definition' and 'practice' of DOT could be discussed separately. Participants' understanding of DOT explored in the current study corroborates the notion that DOT is merely a directly observed and supervised drug swallowing the application of which requires great caution. ${ }^{3540} 41$ The definition of DOT provided by our participants as a medical practice whereby TB patients take their drugs with the attendance of a care provider, coincides with existing understanding of DOT. For example, the WHO mentions that DOT means 'watching patients taking their medications' ${ }^{42}$ and, likewise, Ethiopia's national TB programme ${ }^{34}$ states: 'one of the most important components of DOTS is the direct observation of treatment, which means that a health worker must watch the patient taking each dose'. On the other hand, our participants' understanding of the practice of DOT in Ethiopia differs from that which the WHO recommends. The participants in the current study asserted that DOT, unless compromised, does not have built-in flexibility and rigidly focusses on pill-swallowing. As the purpose of DOT is to ensure that patients swallow drugs,${ }^{43-45}$ it is easy to overlook the patient context unless the healthcare provider chooses to empathise with the patient by, for example, posing such question: Who knows what the patient eats before swallowing the DR-TB tablets? What if it is unacceptable for the patient to take the tablets on some days? How much sacrifice does the patient pay to commute to a healthcare centre every morning? In the eyes of the suffering patient, could adhering to DR-TB treatment be worse than death?

On the contrary, the WHO indicates that sensitivity to patient needs and flexibility-for example, where the TB patient receives treatment-are integral parts of DOT practice. ${ }^{42}{ }^{46}$ However, the $\mathrm{WHO}^{46}$ states that such flexibility is subject to some stipulations: (1) a treatment supporter or observer, whom the national TB control programme is responsible to train and monitor, must be present when the TB patient takes the drugs; (2) 'the drugs should remain with the treatment supporter and be given to the patient only at the time of ingestion'. Similarly, Ethiopia's 'national TB programme recommends supervision of treatment to be made by a trained health worker, health extension worker or a trained TB treatment supporter'. ${ }^{47}$ This evidence shows that the burden of TB treatment should not fall on the patient alone, but rather should be shared. However, sharing this burden-for example, identifying and training treatment supporters for each patient to take treatments at home, or ensuring family and community support to the 
TB patient-requires resources that pose a challenge in resource-poor countries such as Ethiopia. ${ }^{34} 4849$ In the same vein, the following text from the national TB programme provides an insight into the challenges in DOT practice as explored in the current study.

During the initial phase of treatment, which always contains rifampicin, the patient must take the drugs in front of the health worker who is responsible for verifying that the patient swallows all of the prescribed drugs every day. If the patient lives, or can be housed, near a TB clinic, he or she must attend every morning to take the drugs. If the patient lives near a health post with staff that are trained and acknowledged to be capable by the TB clinic coordinator, treatment can be delivered by this health post staff; the follow-up of the patient must continue to be done by the TB clinic, and health post staff must be closely supervised. ...If directly observed treatment cannot be provided on an outpatient basis, or if the condition of the patient requires it, the patient should be hospitalised during the whole of the initial phase of treatment, but this is quite costly. ${ }^{34}$

The accounts of DOT that emerged from the findings show that rigidity in the practice of DOT could serve against the very purpose DOT was invented to accomplish. This finding accords with what Van Deun and Rieder ${ }^{40}$ argue: 'DOT when blindly and carelessly applied, for example, obliging the patient to attend a clinic daily just to be watched while swallowing his/her drugs, will often have the opposite effect'. Moreover, our study coincides with, and responds to, a similar study conducted in India, which questions the necessity of DOT as a pillar of DR-TB treatment and indicates the need to further study the 'validity' of DOT in other settings. ${ }^{45}$ On the contrary, the findings of the current study do not support a systematic review of retrospective observational cohort studies ${ }^{46}$ which indicates that DOT might lead to MDR-TB treatment success. This difference could be, among other potential explanations, due to the fact that the current study exclusively used a qualitative approach to explore subjective experiences.

This study shows that the relationship between patients and healthcare providers is comparable to that found in a family. As DR-TB is a chronic condition, healthcare providers and patients spend longer periods together and as a result, disease and biography merge into each other. In relation to this, we have also identified a high level of empathy characterising the way healthcare providers communicate with, and treat, DR-TB patients. The participants argued that counselling and health education-which they believe are not part of the DOT strategy-are very important to provide effective treatment to DR-TB patients. This relates to the nurses in Peru who reported that emotional support and counselling to MDR-TB patients is necessary to cure them. ${ }^{50}$ Due to the high level of empathy, healthcare providers find it easy to understand their patients when they refuse to adhere to treatments. This finding contradicts the conventional treatment modality in which a significant space between the health professional and the patient exists. Such positive relationships explored in our study result in a better, patient-centred and flexible DOT practice. The findings are contrary to some literature ${ }^{3351}$ which state that healthcare providers lose sight of patient context due to DOT. However, our study did not include DR-TB patients' perspectives and, therefore, the findings cannot be extrapolated to patients. Nonetheless, the findings support evidence from previous studies ${ }^{43} 495253$ which assert that the rigidity of DOT burdens patients, negatively affects patient adherence and worries healthcare providers, thereby negatively affecting TB treatment and control.

The healthcare providers in this study stated that they empathise with DR-TB patients and tinker with DOT to provide health service that they believe is patient-centred. This finding is in line with other studies ${ }^{45555}$ that present healthcare practices in which healthcare providers do not strictly implement treatment guidelines. An effective DR-TB treatment, according to the participants, requires placing DOT in context and finding the most suitable arrangement that empowers the DR-TB patient. This reflects what Mol et $a l^{56}$ mean by 'perfect' care and 'good' care: the perfect care is given 'without considering the world in which the person lives' whereas the good care is 'an arrangement of people and things that is a compromise'. Providing 'perfect care' focusses on impeccably complying with guidelines whereas providing 'good care' focusses on the patient. The results show that improvising medicine could happen due to different reasons. First, healthcare providers tinker in the absence of enough resources and reading materials in place, including global and national DR-TB guidelines, to update and improve their clinical knowledge of DR-TB. Thus, improvisation becomes an 'imposed' practice; it becomes a must. ${ }^{5758}$ Second, on the other hand, healthcare providers tinker because they object to the idea of accepting and venerating DOT as a pillar strategy. Regardless of the availability of resources, healthcare providers chose to place DR-TB patients at the centre of the entire healthcare concept and accordingly adjust every action of care. Thus, the practice of tinkering becomes an 'initiated' practice. ${ }^{59}$ Rather than being the last option, tinkering is an important and a preferable practice for a better treatment outcome. In fact, this is a good opportunity for the Ethiopian government to elevate the fight against DR-TB, optimising the healthcare providers' efforts and enthusiasm for improved care. Resources on DR-TB, including training for healthcare providers, need more attention from government and global partners more than ever to tackle this deadly disease.

One strength of this study was the use of a qualitative methodology to explore healthcare providers' perspectives on the practice of DOT in DR-TB treatment in the study setting. By employing two qualitative data collection methods (IDI and FGD), it attempted to provide 
a comprehensive investigation of the topic. Recruiting participants from both primary- and tertiary-level healthcare facilities contributed to having a wide perspective on the topic. The use of inductive coding facilitated an in-depth understanding of the research question. Moreover, the data collector is from the same cultural background as that of the participants and the participants' working language is his mother tongue, thereby making participants more open to discussions. This study has also limitations. Following a qualitative approach, the participants were purposively selected and there might be selection bias as a result. In addition, data were collected during work hours and this might have limited chances of having longer discussions and richer data.

\section{CONCLUSION}

This study explored healthcare providers' perspectives on the daily practice of DOT and the challenges therein. It has shown that there is a need to embrace tinkering in DR-TB treatment and thereby enhance the practice of DOT among healthcare providers. If rigidly implemented, DOT could lead to more DR-TB - a problem DOT was invented to fight. The findings have significant implications for the rethinking of rigidly implementing guidelines. The findings of this research could be of interest to scholars involved in challenging and questioning mainstream approaches to healthcare and in echoing the voices of health workers, thus emphasising the need to incorporate their perspectives in designing healthcare guidelines and policies. It is high time to shape the practice of DOT for DR-TB patients. More research drawing perspectives from disciplines such as medical ethics, human rights and anthropology could further explore the challenges in integrating and implementing DOTS for DR-TB within fragile healthcare systems wherein resources needed to ensure a patient-centred treatment are limited. We suggest that more DOT training should be given to healthcare practitioners, in line with their specific role in DR-TB care, to bring about positive changes. The Ethiopian government needs to strengthen its collaboration with global partners to leverage greater resources needed for the DR-TB care and treatment programme.

\section{Author affiliations}

${ }^{1}$ Department of Community Medicine and Global Health, Institute of Health and Society, University of Oslo, Oslo, Norway

${ }^{2}$ Institute for Biomedical Ethics, University of Basel, Basel, Switzerland

${ }^{3}$ Addis Ababa University, College of Health Sciences, Center for Innovative Drug Development and Therapeutic Trials for Africa, Addis Ababa, Ethiopia

Acknowledgements The authors wish to acknowledge all the healthcare workers and health centres that participated in this study, as well as the Addis Ababa City Administration Health Bureau.

Contributors All authors (KMM, CG and TM) were involved in study conception. KMM was involved in data acquisition. All authors (KMM, CG and TM) were involved in data analysis. KMM and TM wrote the first draft. All authors (KMM, CG and TM) reviewed the paper, provided comments and approved the final version.

Funding This work was supported in part by the Institute of Health and Society, University of Oslo, Norway (author Kirubel Manyazewal Mussie) and the Fogarty
International Center and National Institute of Allergy and Infectious Diseases of the U.S. National Institutes of Health under award number D43TW009127 (author Dr Tsegahun Manyazewal).

\section{Competing interests None declared.}

Patient and public involvement Patients and/or the public were not involved in the design, or conduct, or reporting or dissemination plans of this research.

\section{Patient consent for publication Not required.}

Ethics approval Ethical clearance for the study was obtained from the Norwegian Centre for Research Data, Norway. Moreover, the study was given ethical approval by Addis Ababa City Administration Health Bureau in Ethiopia.

Provenance and peer review Not commissioned; externally peer reviewed.

Data availability statement No data are available. All data relevant to the study are included in the article or uploaded as supplementary information.

Open access This is an open access article distributed in accordance with the Creative Commons Attribution Non Commercial (CC BY-NC 4.0) license, which permits others to distribute, remix, adapt, build upon this work non-commercially, and license their derivative works on different terms, provided the original work is properly cited, appropriate credit is given, any changes made indicated, and the use is non-commercial. See: http://creativecommons.org/licenses/by-nc/4.0/.

\section{ORCID iDs}

Kirubel Manyazewal Mussie http://orcid.org/0000-0002-9313-1186

Tsegahun Manyazewal http://orcid.org/0000-0002-8360-7574

\section{REFERENCES}

1 Paciej-Gołębiowska P, Pikala M, Maniecka-Bryła I. Years of life lost due to diseases of the digestive system in Poland according to socioeconomic factors: a cross-sectional study. BMJ Open 2019;9:e030304.

2 Thompson R, Garry S. The political economy of NCDS, and the limits of global health. Lancet 2019;393:1186-7.

3 Van Malderen C, Amouzou A, Barros AJD, et al. Socioeconomic factors contributing to under-five mortality in sub-Saharan Africa: a decomposition analysis. BMC Public Health 2019;19:760.

4 Barnish M, Tørnes M, Nelson-Horne B. How much evidence is there that political factors are related to population health outcomes? an internationally comparative systematic review. BMJ Open 2018;8:e020886.

5 Dahab M, Abdelmagid N, Osama T, et al. Political violence in Sudan: the need for a coordinated, locally led humanitarian health response. Lancet 2019;394:549-51.

6 Friis Abrahamsen C, Ahrensberg JM, Vedsted P. Utilisation of primary care before a childhood cancer diagnosis: do socioeconomic factors matter?: a Danish nationwide population-based matched cohort study. BMJ Open 2018;8:e023569.

7 Sakamoto $\mathrm{H}$, Lee S, Ishizuka A, et al. Challenges and opportunities for eliminating tuberculosis - leveraging political momentum of the UN high-level meeting on tuberculosis. BMC Public Health 2019;19:76.

8 WHO. Ten threats to global health in 2019 Geneva, Switzerland: who, 2019. Available: https://www.who.int/vietnam/news/feature-stories/ detail/ten-threats-to-global-health-in-2019

9 Dheda K, Gumbo T, Maartens G, et al. The Lancet respiratory medicine Commission: 2019 update: epidemiology, pathogenesis, transmission, diagnosis, and management of multidrug-resistant and incurable tuberculosis. Lancet Respir Med 2019;7:820-6.

10 Lange C, Dheda K, Chesov D, et al. Management of drug-resistant tuberculosis. Lancet 2019;394:953-66.

11 Singh, Dwivedi, Gaharwar. Recent updates on drug resistance in Mycobacterium tuberculosis 2019.

12 Alele FO, Franklin RC, Emeto TI, et al. Occupational tuberculosis in healthcare workers in sub-Saharan Africa: a systematic review. Arch Environ Occup Health 2019;74:95-108.

13 Aliyu G, El-Kamary SS, Abimiku Alash'le, et al. Demography and the dual epidemics of tuberculosis and HIV: analysis of cross-sectional data from sub-Saharan Africa. PLoS One 2018;13:e0191387.

14 Chem ED, Van Hout MC, Hope V. Treatment outcomes and antiretroviral uptake in multidrug-resistant tuberculosis and HIV co-infected patients in sub Saharan Africa: a systematic review and meta-analysis. BMC Infect Dis 2019;19:723.

15 Mohammed H, Assefa N, Mengistie B, et al. Prevalence of extrapulmonary tuberculosis among people living with HIV/AIDS in 
sub-Saharan Africa: a systemic review and meta-analysis. Hiv Aids 2018;10:225-37.

16 Ngabonziza JCS, Diallo AB, Tagliani E, et al. Half of rifampicinresistant Mycobacterium tuberculosis complex isolated from tuberculosis patients in sub-Saharan Africa have concomitant resistance to pyrazinamide. PLoS One 2017;12:e0187211.

17 Wild V, Jaff D, Shah NS, et al. Tuberculosis, human rights and ethics considerations along the route of a highly vulnerable migrant from sub-Saharan Africa to Europe. Int J Tuberc Lung Dis 2017;21:1075-85.

18 WHO. Global tuberculosis report 2018. Geneva, 2018.

19 World Health Organization. The end TB strategy: global strategy and targets for tuberculosis prevention, care and control after 2015. Geneva, Switzerland, 2015.

20 Bynum H. Spitting blood: the history of tuberculosis. Oxford: Oxford University Press, 2012.

21 Davies PDO. The role of dots in tuberculosis treatment and control. Am J Respir Med 2003;2:203-9.

22 Gradmann C. Treatment on trial: Tanzania's national tuberculosis program, the International Union against tuberculosis and lung disease, and the road to dots, 1977-1991. J Hist Med Allied Sci 2019;74:316-43.

23 Espinal MA, Kim SJ, Suarez PG, et al. Standard short-course chemotherapy for drug-resistant tuberculosis: treatment outcomes in 6 countries. JAMA 2000;283:2537-45.

24 Farmer P, Kim JY. Community based approaches to the control of multidrug resistant tuberculosis: introducing "DOTS-plus". British Medical Journal Publishing Group 1998;671.

25 WHO. DOTS-Plus \& Green Light Committee: Improving access to second-line anti-TB drugs. Geneva, Switzerland, 2000.

26 Laing RO, McGoldrick KM, Laing RO. Tuberculosis drug issues: prices, fixed-dose combination products and second-line drugs. Int $J$ Tuberc Lung Dis 2000;4:S194-207.

27 Ollé-Goig JE. Control of multidrug resistant tuberculosis. DOTS-plus strategy will be hard to implement. BMJ 1999;318:736-36.

28 Van RieA, Warren R, Mshanga I, et al. Analysis for a limited number of gene codons can predict drug resistance of Mycobacterium tuberculosis in a high-incidence community. J Clin Microbiol 2001;39:636-41.

29 Bastian I, Stapledon R, Colebunders R. Current thinking on the management of tuberculosis. Curr Opin Pulm Med 2003;9:186-92.

30 Sterling TR, Lehmann HP, Frieden TR. Impact of dots compared with DOTS-plus on multidrug resistant tuberculosis and tuberculosis deaths: decision analysis. BMJ 2003;326:574.

31 Gupta R, Espinal M, Stop TB Working Group on DOTS-Plus for MDR-TB. A prioritised research agenda for DOTS-Plus for multidrug-resistant tuberculosis (MDR-TB). Int $J$ Tuberc Lung Dis 2003;7:410.

32 Nathanson E, Gupta R, Huamani P, et al. Adverse events in the treatment of multidrug-resistant tuberculosis: results from the DOTSPlus initiative. Int J Tuberc Lung Dis 2004;8:1382-4.

33 Harper I. Extreme condition, extreme measures? compliance, drug resistance, and the control of tuberculosis. Anthropol Med 2010;17:201-14.

$34 \mathrm{FMoH}$. National comprehensive tuberculosis, leprosy and TB/HIV training manual for health care workers. Addis Ababa, Ethiopia: Ethiopian Federal Ministry of Health (FMoH), 2016.

35 Moonan PK, Quitugua TN, Pogoda JM, et al. Does directly observed therapy (dot) reduce drug resistant tuberculosis? BMC Public Health 2011;11:19.

36 Dicicco-Bloom B, Crabtree BF. The qualitative research interview. Med Educ 2006;40:314-21.

37 Kielmann KC. Introduction to qualitative research methodology. Enttebe, Uganda: Evidence for Action Research Programme Consortium, 2010: 84.
38 Guest G, Namey E, Taylor J, et al. Comparing focus groups and individual interviews: findings from a randomized study. Int J Soc Res Methodol 2017;20:693-708.

39 Liamputtong P, Braun V, Clarke V, et al. Thematic analysis 2018.

40 Van Deun A, Rieder HL. Dot, S, or dots? Public Health Action 2012;2:3.

41 Zwarenstein M, Schoeman JH, Vundule C, et al. Randomised controlled trial of self-supervised and directly observed treatment of tuberculosis. Lancet 1998;352:1340-3.

42 Organization WH. What is dots? A guide to understanding the WHO-recommended TB control strategy known as dots. Geneva, Switzerland: WHO, 1999.

43 Hilawe AM, Teshale F D. Community perception to directly observed treatment short course among tuberculosis patients in Bahirdar, North Ethiopia (a qualitative study). Journal of Biology, Agriculture and Healthcare 2016;6.

44 WHO. Companion Handbook to the who guidelines for the programmatic management of drug-resistant tuberculosis. Geneva: WHO Press, 2014.

45 Benbaba S, Isaakidis P, Das M, et al. Direct observation (do) for drug-resistant tuberculosis: do we really do? PLoS One 2015;10:e0144936.

46 Orenstein EW, Basu S, Shah NS, et al. Treatment outcomes among patients with multidrug-resistant tuberculosis: systematic review and meta-analysis. Lancet Infect Dis 2009;9:153-61.

47 Ethiopia FMoH. Guidelines for management of TB, DR-TB and leprosy in Ethiopia. Sixth ed. Addis Ababa, Ethiopia Federal Ministry of Health Ethiopia, 2018.

48 Gebreegziabher SB, Yimer SA, Bjune GA. Qualitative assessment of challenges in tuberculosis control in West Gojjam zone. Northwest Ethiopia: Health Workers' and Tuberculosis Control Program Coordinators' Perspectives, 2016.

49 Sagbakken M, Frich JC, Bjune G. Barriers and enablers in the management of tuberculosis treatment in Addis Ababa, Ethiopia: a qualitative study. BMC Public Health 2008;8:11.

50 Chalco K, Wu DY, Mestanza L, et al. Nurses as providers of emotional support to patients with MDR-TB. Int Nurs Rev 2006;53:253-60.

51 Sagbakken M. Tuberculosis as a global challenge : a qualitative study of patients' and health workers' perception and management of tuberculosis in Ethiopia and Norway 2010.

52 Pinto LM, Udwadia ZF. Private patient perceptions about a public programme; what do private Indian tuberculosis patients really feel about directly observed treatment? BMC Public Health 2010;10:357-57.

53 Tadesse T, Demissie M, Berhane $\mathrm{Y}$, et al. Long distance travelling and financial burdens discourage tuberculosis dots treatment initiation and compliance in Ethiopia: a qualitative study. BMC Public Health 2013;13:424.

54 Dunn MC, Clare ICH, Holland AJ. Living 'a life like ours': support workers' accounts of substitute decision-making in residential care homes for adults with intellectual disabilities. J Intellect Disabil Res 2010:54:144-60.

55 Horn R. "Why Should I Question a Patient's Wish?"A Comparative Study on Physicians' Perspectives on Their Duties to Respect Advance Directives. Eur J Health Law 2017;24:523-40.

$56 \mathrm{Mol}$ A, Moser I, Pols J. Care in practice : on tinkering in clinics, homes and farms. Bielefeld: Transcript Verlag, 2010.

57 Livingston J. Improvising medicine : an African oncology ward in an emerging cancer epidemic. Durham, N C: Duke University Press, 2012.

58 Prince RJ, Otieno P. In the Shadowlands of global health: observations from health workers in Kenya, 2014.

59 McKenna K, Leykum LK, McDaniel RR. The role of Improvising in patient care. Health Care Manage Rev 2013;38:1-8. 